\title{
Geometrical and thermal influences on a bacterial cellulose-based sensing element for acceleration measurements
}

\author{
Giovanna Di Pasquale ${ }^{1}$, Salvatore Graziani ${ }^{2}$, Antonio Licciulli ${ }^{3}$, Rossella Nisi ${ }^{4}$, Antonino Pollicino5 \\ Carlo Trigona ${ }^{2}$ \\ ${ }^{1}$ D.S.C., Dipartimento di Scienze Chimiche, University of Catania, Viale Andrea Doria 6,95125, Catania, Italy \\ 2 D.I.E.E.I., Dipartimento di Ingegneria Elettrica Elettronica e Informatica, University of Catania, Viale Andrea Doria 6, 95125, Catania, Italy \\ 3 Dipartimento di Ingegneria dell'Innovazione, University of Lecce, Via per Arnesano, 73100, Lecce, Italy \\ ${ }^{4}$ BioFaber, via Luigi di Savoia 19, Mesagne, Brindisi, Italy \\ ${ }^{5}$ D.I.C.A.R., Dipartimento di Ingegneria Civile e Architettura, University of Catania, Viale Andrea Doria 6, 95125, Catania, Italy
}

\begin{abstract}
The need for a sustainable economy necessitates new environmentally friendly production technologies as well as devices that can be easily recycled, disposed of, and, finally, degraded, without any release of pollutants to the environment. In this context, bacterial cellulose (BC) has recently been investigated as an intriguing solution for the creation of green motion sensors. BC has excellent mechanical properties, and it is fully biodegradable and greener than the more common plant-derived cellulose. In this paper, we investigate the influence of geometry and environmental temperature on BC-based sensing elements. More specifically, the influence of these quantities on a previously investigated BC-based accelerometer are reported. An experimental campaign and the characterization of the proposed green device for several geometries (from 7 to $22 \mathrm{~mm}$ of length) and various temperatures (from $5{ }^{\circ} \mathrm{C}$ to $55^{\circ} \mathrm{C}$ ) is addressed, obtaining very intriguing results.
\end{abstract}

\section{Section: RESEARCH PAPER}

Keywords: bacterial cellulose; ionic liquids; greener transducers; biodegradable sensor; geometry analysis

Citation: Carlo Trigona, Giovanna Di Pasquale, Salvatore Graziani, Antonino Licciulli, Rossella Nisi, Antonino Pollicino, Carlo Trigona, Geometrical and thermal influences on a bacterial cellulose based sensing element for acceleration measurements for acceleration measurements, Acta IMEKO, vol. 9, no. 4, article 20, December 2020, identifier: IMEKO-ACTA-09 (2020)-04-20

Section Editor: Francesco Bonavolonta, University of Naples Federico II, Italy

Received October 31, 2019; In final form March 4, 2020; Published December 2020

Copyright: @2020 IMEKO. This is an open-access article distributed under the terms of the Creative Commons Attribution 3.0 License, which permits unrestricted use, distribution, and reproduction in any medium, provided the original author and source are credited.

Corresponding author: Carlo Trigona, e-mail: carlo.trigona@dieei.unict.it, Salvatore Graziani, e-mail: salvatore.graziani@dieei.unict.it

\section{INTRODUCTION}

The introduction of digitalisation in virtually all aspects of everyday life is revolutionising people's lifestyles. Not less important, new ecologies, such as the Internet of Things (IoT) and Industry 4.0, require the mass-production of low-cost electronic devices to implement new functionalities in unexplored environments and working conditions. As a result, vigorous growth in the economy can be envisaged both in developed and developing countries, with a corresponding beneficial effect on people's wealth. Unfortunately, side effects need to be considered in such a promising scenario. The trend for the lifetime of electronics is significantly decreasing; a cell phone is used for less than 18 months before being replaced, a computer for less than 3 years.

In 2007, 3.2 million tons of e-waste were generated in the US, with a corresponding increase of landfilling and environmental concerns [1]. New production strategies are required that are capable both of saving nonrenewable resources and of producing electronics that do not create environmental concerns after their End of Life (EOL). As it stands, existing technologies for the manufacture of integrated circuits (IC) use hazardous substances that, if not suitably processed, can pose significant risks to human health. This problem is leading to significant challenges, especially in developing countries where rudimentary treating procedures are implemented [2]. 
Low-power, renewable production processes and easily biodegradable materials that do not pollute the environment after disposal are required to create a more sustainable economy. Though polymers have attracted the interest of the scientific community because they can introduce new functionalities, such as affordability, flexibility, and biocompatibility, they are not biodegradable, and concerns exist about the treatment of polymer-based electronics after their EOL. Cellulose, the most widely available biopolymer on the earth, looks to be a suitable candidate for producing next-generation electronics. It is cheap, biocompatible, and biodegradable. Additionally, cellulose and its derivatives have interesting mechanical and electrical properties that have been exploited in many applications [3]-[6]. Notwithstanding such promising properties, plant-derived cellulose extraction by the pulp industry involves significant resource consumption in terms of energy and fresh water. According to the EU, the average energy costs for the pulp and paper industry equal around $16 \%$ of production costs. $38 \%$ of the energy used comes from natural gas [7]. Not less important, pollutants are produced by the pulp and paper industry, raising concerns about their environmental effects.

Bacterial cellulose (BC) is a kind of cellulose produced by some bacteria genera when maintained in suitable culturing environments. In this case, the cellulose is directly produced by the bacteria at the interface between the growing media and the open air. The production process can be implemented in typical laboratory conditions and enables the production of very pure cellulose. BC has been proposed for manufacturing electronics and sensors [8], [9]. Recently, BC has also been proposed for the manufacture of both actuators and hybrid and conventional solutions for mechanical sensors [10], [11].

The possibility of using BC-based composites for the manufacture of green sensors has been investigated [12]-[13]. Composites consisting of a bulk BC membrane impregnated with ionic liquids (IL) and covered on both sides by poly(3,4-ethylenedioxythiophene) polystyrene sulfonate (PEDOT:PSS) have been fabricated.

In particular, the inertial- and mass-sensing properties of such composites when used in a cantilever configuration have been investigated [12]-[14]. Moreover, the influence of geometry on the sensing properties of an accelerometer has been analysed [12], [15].

Polymer composites are known to be sensitive to environmental parameters, temperature and relative humidity being the most influential [16], [17]. Based on such considerations, the present paper carries the investigation of the accelerometer further, by investigating the influence of environmental temperature on the transducing properties of the $\mathrm{BC}$-based composites. The influence of temperature on the investigated $\mathrm{BC}$-based composites is even more relevant because of the presence of ILs, which can have freezing points that are similar to the environmental temperature. Results reported here complete the investigation reported in our previous study, and finally provide evidence of the role ILs play in the transduction properties of the $\mathrm{BC}$ composites we investigated [15]. The results we obtained contribute to a better understanding of the performance of such a new composite, a necessary task ahead of its use in real sensing problems.

The paper is organised as follows: Section 2 describes the materials and the preparation procedure. Section 3 reports the characterisation setup. Section 4 shows the results, and the conclusions are given in Section 5.

\section{MATERIALS AND PREPARATION PROCEDURE}

The following commercial products were explored to make the $\mathrm{BC}$ sensing element:

- Black tea bags

- Sucrose

- Vinegar

- Kombucha tea

Furthermore, sodium hydroxide $(\mathrm{NaOH})$ and absolute ethanol $(99.8 \%)$ were purchased from Sigma-Aldrich and used without further modification. PEDOT:PSS and CLEVIOS PHCV4 were purchased from H.C. Starck for aqueous dispersion. EMIM-BF 4 , an IL, was purchased from Alfa Aeser.

Concerning the preparation procedure for the compound, $\mathrm{BC}$ pellicles were obtained from the fermentation process of kombucha tea, a sweetened black tea with Acetobacter strains [18]. The bacterial culture was composed of upper cellulosic pellicle and a lower liquid broth [19].

The culture medium was prepared by adding $70 \mathrm{~g}$ of sucrose and $4 \mathrm{~g}$ of black tea to 11 of boiling water. Then the mixture was left to steep for $15 \mathrm{~min}$ and, after removing the tea, the $\mathrm{pH}$ value of the broth was adjusted to $2.7-3.0$ by adding $10 \mathrm{ml}$ of acetic acid for each liter of broth. The addition of acetic acid at the beginning of the fermentation process prevents the formation of molds and protects against undesirable microorganisms. Finally, the cellulosic pellicle pieces $(3 \% \mathrm{w} / \mathrm{v})$ and liquid broth $(10 \% \mathrm{v} / \mathrm{v})$ with the Acetobacter strains $(1 \mathrm{ml} / \mathrm{l})$ were added to the cooled tea broth. A Teflon stopper was attached to the lower part of the beaker to block the pellicle.

In the initial stage, the bacteria increase their population by consuming dissolved oxygen and producing a certain amount of liquid cellulose, as observed by the appearance of turbidity. After that, a pellicle of cellulose is grown on the surface of the broth.

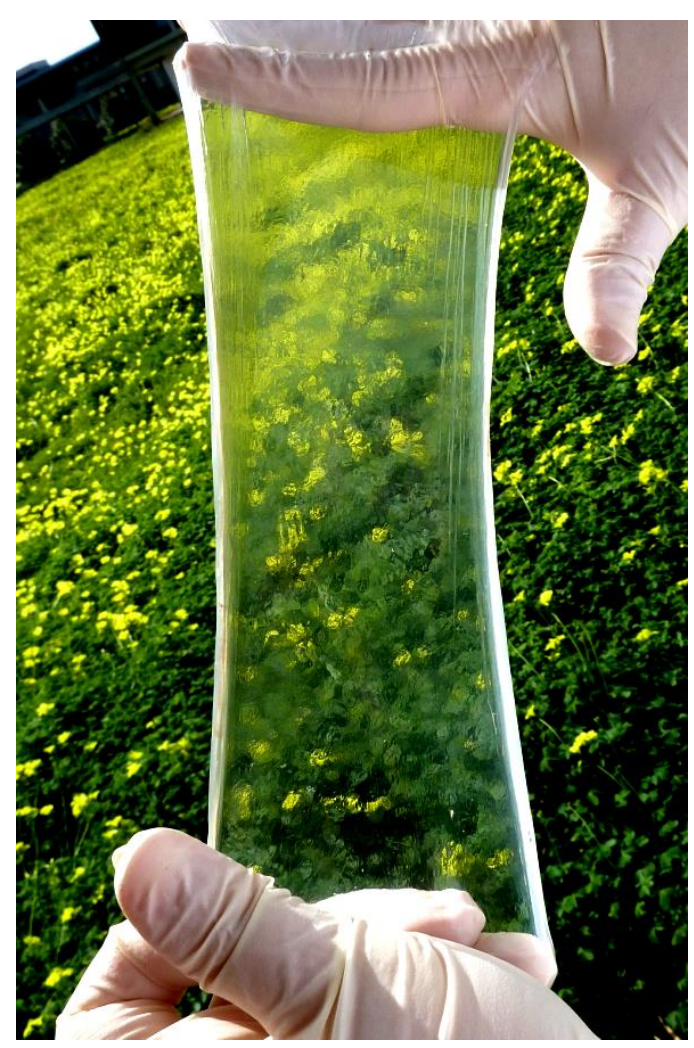

Figure 1. Picture of the prepared $\mathrm{BC}$, without contacts and before the drying procedure. 
When the dissolved oxygen is used up, only bacteria existing near the surface can maintain their activity and produce cellulose. This medium was used to inoculate new fermentations. The pellicles of nanofibrillated $\mathrm{BC}$ were thoroughly washed with distilled water and boiled in $0.5 \mathrm{M} \mathrm{NaOH}$ solution for $2 \mathrm{~h}$ in order to remove all impurities, such as the medium or endotoxins from the $\mathrm{BC}$ samples, contained in the cell wall of the bacteria. The pellicles of $\mathrm{BC}$ were then washed in water 4 times. Figure 1 shows the $\mathrm{BC}$ after production.

The $\mathrm{BC}$ was dried in an oven at $80^{\circ} \mathrm{C}$ overnight to eliminate the water content. Upon retrieving the samples, they were further dehydrated in an oven to eliminate any water absorbed during the storage phase. The samples were then cut, infused with EMIM-BF 4 as the IL, and covered with PEDOT:PSS as flexible electrodes.

\section{CHARACTERISATION SETUP}

In order to analyse the sensor as a function of its geometry and in terms of the external temperature, a suitable experimental setup was conceived. In particular, Figure 2 shows the entire block diagram used for the study and the characterisation. Figure 3 shows the experimental setup used to characterise the system. A temperature-controlled chamber was used for the thermal characterisation of the device. The experimental setup consisted of:

- A shaker to excite the structure

- An HP33120A signal generator to produce a sinusoidal waveform

- A digital Teledyne LeCroy WaveRunner 6050 to measure the signals

- An inertial sensor to monitor the level of acceleration imposed through the shaker

- A temperature-controlled chamber to investigate the effect of temperature

- A B2985A Electrometer/High Resistance Meter to measure the resistance and the output current of the sensing element

Figure 4 shows a sample of the BC-based compound. The black surface is the PEDOT:PSS layer.

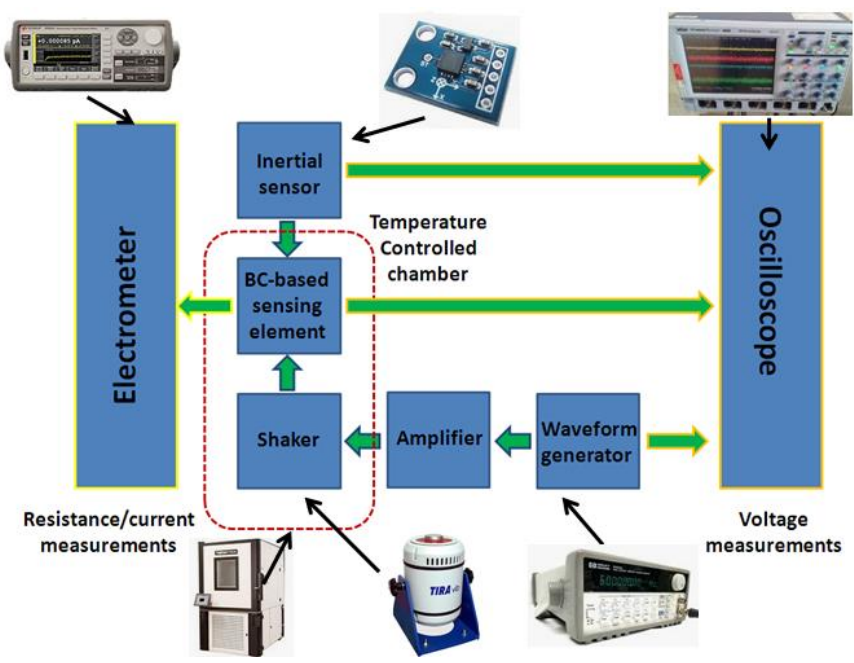

Figure 2. Block diagram of the equipment used to characterise the BC-based sensing element.

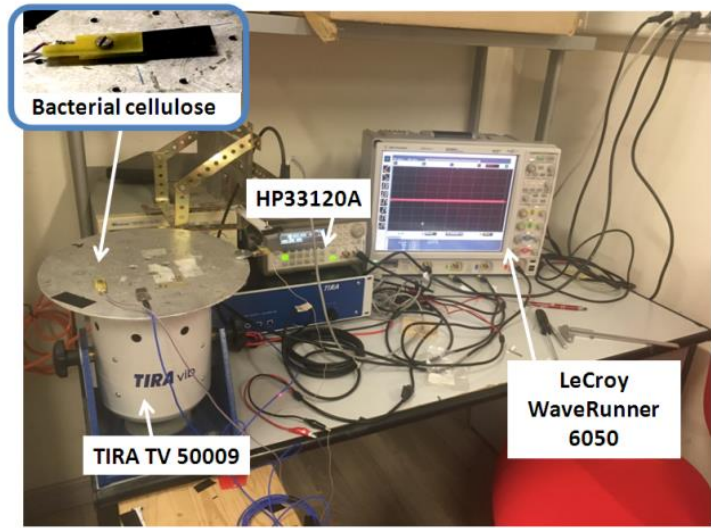

Figure 3. Experimental setup. The inset shows a sample of the BC-based compound mounted between two rigid electrodes, which were used to detect the electrical signal that was generated.

\section{EXPERIMENTAL RESULTS}

The measurement campaign was composed of two parts. The first investigation (subsection 4.1) compared the characterisation of the sensor for several geometries (from $7 \mathrm{~mm}$ to $22 \mathrm{~mm}$ of length). More specifically, the generated output voltage as a function of the imposed acceleration was examined along with the influence of the transducer length on these relationships.

The second investigation, (subsection 4.2) explored the thermal characterisation of the device, using a temperature range from $5{ }^{\circ} \mathrm{C}$ to $55^{\circ} \mathrm{C}$. The influence of temperature on both static and dynamic working conditions was investigated.

\subsection{Geometrical influences}

The investigation was conducted using samples with a width of $11 \mathrm{~mm}$ and a thickness of about $0.25 \mathrm{~mm}$. Devices with lengths of $22 \mathrm{~mm}, 17 \mathrm{~mm}, 12 \mathrm{~mm}$, and $7 \mathrm{~mm}$ were tested. An analysis of the output voltage as a function of the imposed acceleration was performed in the frequency domain for each transducer geometry. The measurements were obtained by forcing a sinusoidal signal through the signal generator (see Section 3) at the mechanical resonant frequency of each device. It is worth noting that for the four case studies, the following

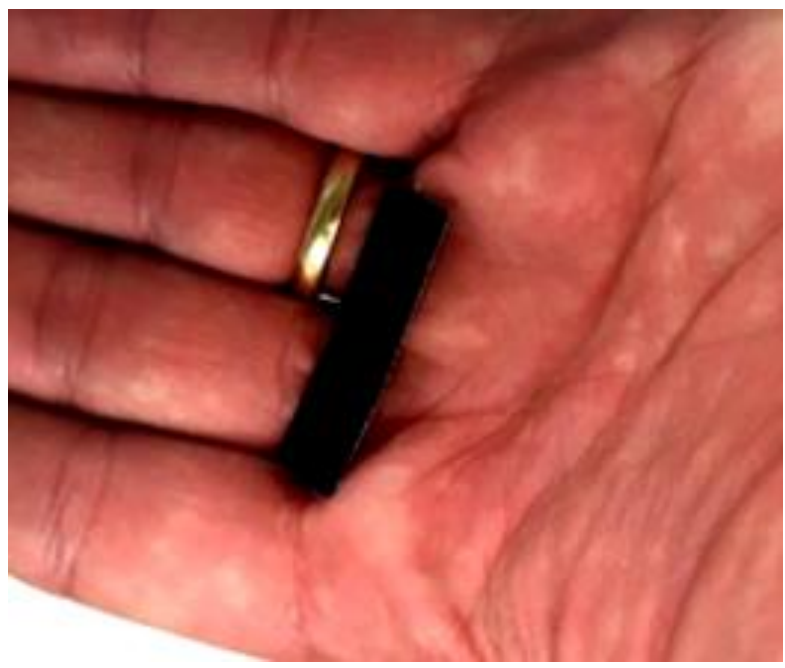

Figure 4. The realised device (length $35 \mathrm{~mm}$, width $11 \mathrm{~mm}$, and thickness about $0.25 \mathrm{~mm}$ ) with the two electrical contacts on the top and bottom faces. The black surface is the PEDOT:PSS layer. The BC-IL composite is sandwiched between the two PEDOT:PSS layers. 
a)

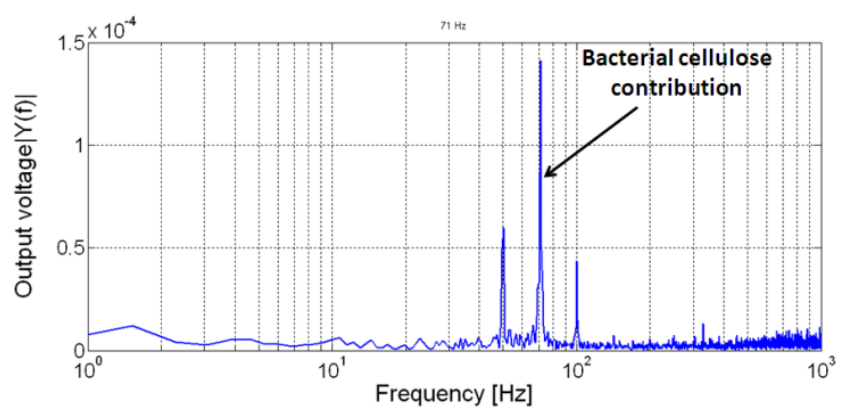

b)

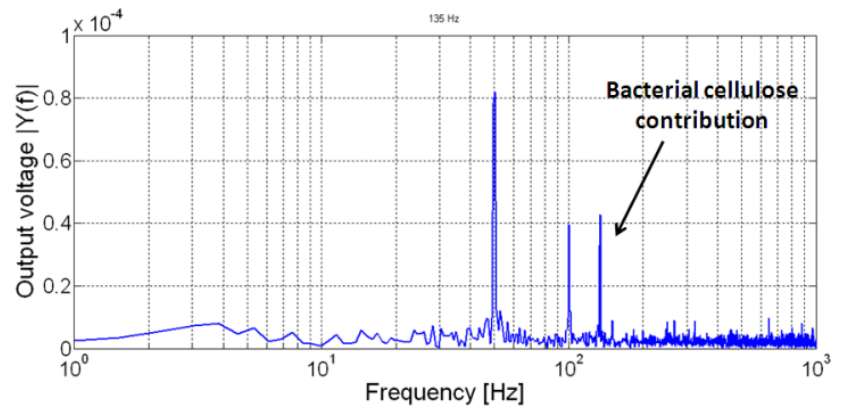

Figure 5. FFT of the output voltage (expressed in V) of a) $\mathrm{BC}$ with a length of $22 \mathrm{~mm}$ (frequency of $71 \mathrm{~Hz}$ ), b) $B C$ with a length of $7 \mathrm{~mm}$ (frequency of $135 \mathrm{~Hz}$ ). Two other spikes can be seen in the figure, indicating the powering line contribution ( $50 \mathrm{~Hz}$ and $100 \mathrm{~Hz}$ contributions, respectively).

mechanical resonant frequency values were experimentally estimated: $71 \mathrm{~Hz}$ (device \#1, length of $22 \mathrm{~mm}$ ), $110 \mathrm{~Hz}$ (device \#2, length of $17 \mathrm{~mm}$ ), $133 \mathrm{~Hz}$ (device \#3, length of $11 \mathrm{~mm}$ ) and $135 \mathrm{~Hz}$ (device \#4, length of $7 \mathrm{~mm}$ ). More details concerning the spectrum of the output voltage are shown in Figure $5 \mathrm{a}$ and Figure 5b, where the FFT of the output for devices \#1 and \#4 are presented, respectively. Figure 6 shows the normalised output voltage as a function of the normalised imposed acceleration. For sake of comparison, the voltage of each sample has been normalised to the output voltage of the first sample, i.e., the device with a length of $22 \mathrm{~mm}$. Furthermore, the acceleration values have been normalised to the acceleration a1, which refers to the first sample. The value a2 is the acceleration level at a frequency of $110 \mathrm{~Hz}$ normalised to a1, and so on for a3 (at 133 $\mathrm{Hz}$ ) and a4 (at $135 \mathrm{~Hz}$ ), respectively. Figure 7 shows the normalised output voltage as a function of the normalised length of the beam. The normalisation has been performed using the length of device \#1.

\subsection{Thermal influences}

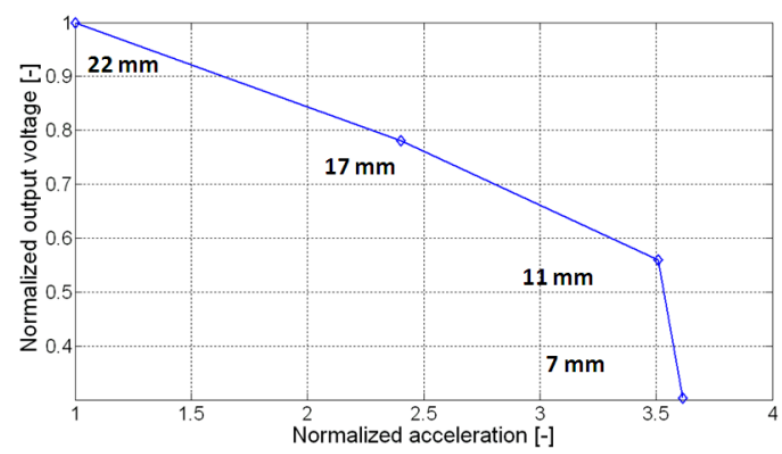

Figure 6. Normalised output voltage as a function of normalised acceleration.

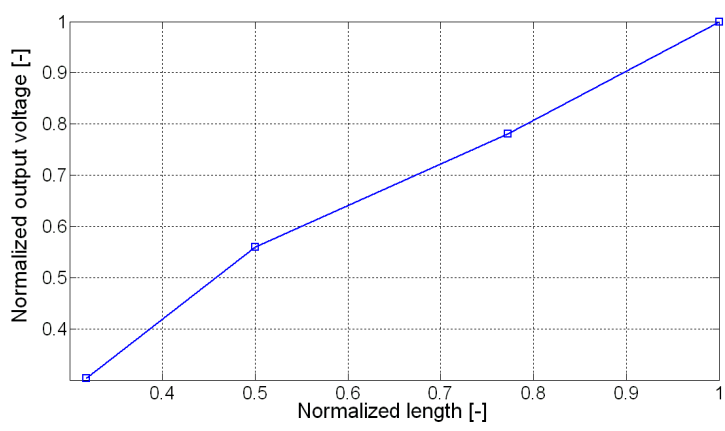

Figure 7. Normalised output voltage as a function of normalised length.

The investigation described in this section was devoted to studying the behaviour of the $\mathrm{BC}$ sensing element in the presence of temperature changes. A device having a geometrical characteristic similar to prototype \#1 (see Section 4.1) was considered to have a significant output voltage.

In particular, two aspects were investigated:

1) The static characterisation was based on an analysis of electrical properties as a function of environmental temperature variation. During this set of experiments, no motion was applied to the device, and the electrical properties of the composite were investigated.

2) The dynamic characterisation was based on the study of the generation capability of the $\mathrm{BC}$ device at various temperatures. In this latter case, the shaker was actuated inside the thermal chamber to mechanically excite the base of the sensing element, which deformed according to its mechanical inertia.

The considered range of temperature was from $5{ }^{\circ} \mathrm{C}$ to $55^{\circ} \mathrm{C}$. The minimum value was selected due to the fact that the IL used for the prototype (see Section 2) has a melting point of about $15{ }^{\circ} \mathrm{C}$ [20]. For this reason, it was interesting to investigate temperatures lower than this value. The upper temperature of $55^{\circ} \mathrm{C}$ was chosen due to the fact that the maximum operative temperature of the device is about $60^{\circ} \mathrm{C}$. This limit is given by the melting temperature of PEDOT:PSS, which was used to make the contacts of the BC-based sensing element.

In order to analyse the influence of temperature on the resistance of the device, an electrometer (see Section 3) was used, and the device was inserted inside the thermal chamber.

Figure 8 shows the resistance of the $\mathrm{BC}$ as a function of the imposed temperatures in the absence of mechanical vibrations (static analysis). Six values were considered. Each temperature value was measured through an internal thermal sensor. Each point in the graph represents the mean value and the measurement uncertainty $(3 \sigma)$.

As it can be noted, a decrement of the resistance across the $\mathrm{BC}$ can be observed for increasing temperature values. The observed effect is, of course, a cumulative effect, reflecting possible changes either in the bulk or at the electrodes. With regard to the electrodes' contribution, the observed phenomenon can be explained by considering the low level of conductivity associated with the PEDOT:PSS electrodes [21]. Nevertheless, further investigation is needed to determine any contribution of the bulk to the decrement of the resulting resistance. Figure 9 shows the voltage across the $\mathrm{BC}$ as a function of time for each of the six temperature values imposed inside the chamber. As it can be observed, the voltage decreases when the temperature value increases. It is worth noting that this behaviour is in accordance with the previous result for resistance. Namely, the electrometer imposes the current, so that the voltage 


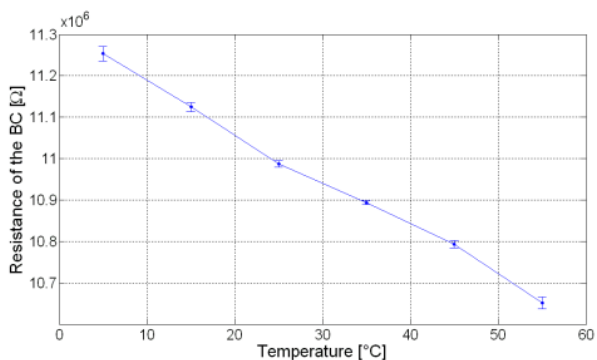

Figure 8. Resistance of the $\mathrm{BC}$ as a function of temperature. The connecting segments are introduced to help the eye.

across the device changes because of the change in the resistance value of the $\mathrm{BC}$.

In order to study the device from a dynamic point of view, it was mechanically actuated by using the shaker and imposing a sinusoidal waveform with a fixed amplitude and frequency (tuned to the mechanical resonance) through the signal generator (see Section 3).

The shaker was inserted inside the chamber. The temperature values were imposed in order to study the changes in the current generated by the $\mathrm{BC}$ as a function of the temperature.

Figure 10 shows the generated current as a consequence of the mechanical vibration for different values of the environmental temperature. A low pass filter was applied in order to decrease the noise level.

As it can be observed, the analysis shows that the generated current is temperature dependent. The output current starts to increase at temperature values larger than $15^{\circ} \mathrm{C}$, which coincides with the melting point of the IL used in the compound. Furthermore, while higher temperature values increase the current, they could have adverse effects on the electrical contacts. For these reasons, the region between $15^{\circ} \mathrm{C}$ and $45^{\circ} \mathrm{C}$ represents an interesting operative range for the $\mathrm{BC}$-based sensing element.

The effect of the freezing temperature of the ionic liquids has been also investigated, as shown in Figure 11.

The graph evinces that when the compound is used at a temperature lower than the meting point of the IL, the output current is close to zero.

It has been widely reported in the literature that in the case of $\mathrm{BC}$ impregnated with ILs and covered with PEDOT:PSS, the electromechanical transduction of the actuators is due both to the IL's migration inside the $\mathrm{BC}$ bulk and electrochemical doping processes at the polymeric electrodes [4], [22]. The results reported here clearly show that the ILs also play a relevant role in the mechanoelectrical transduction properties of $\mathrm{BC}$-based

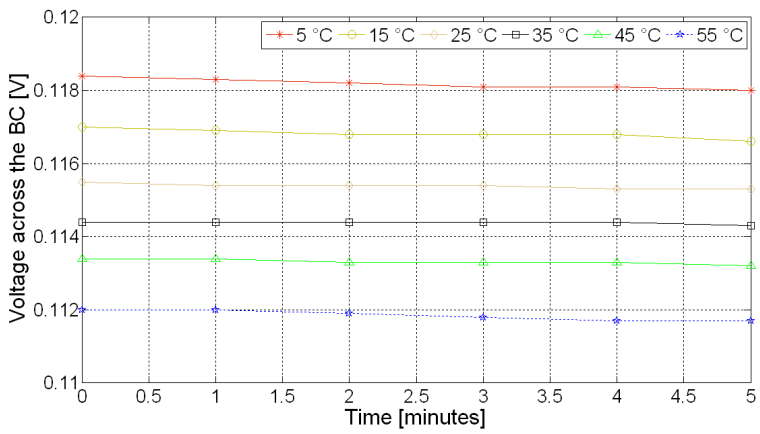

Figure 9. Measurement of the change in voltage across the $B C$ for six temperature values.

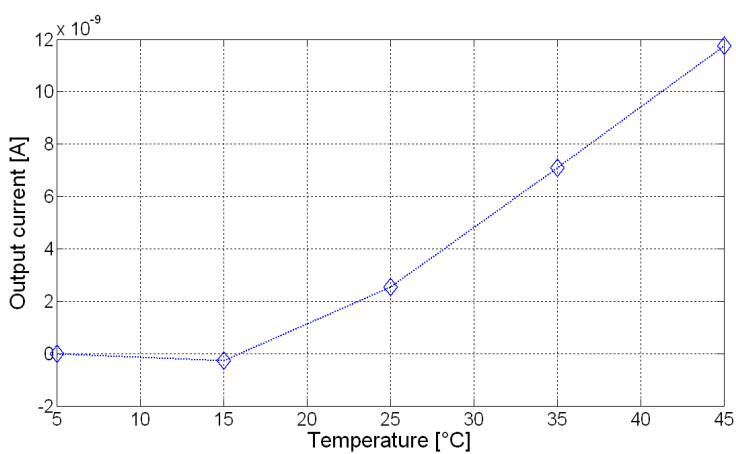

Figure 10. Generated current as a consequence of the mechanical vibration, function of temperature.

composites. In fact, at temperatures lower than the IL's freezing point, the charges inside the $\mathrm{BC}$ compound cannot move. As a consequence, no current can be generated in the presence of mechanical vibrations. To the best of our knowledge, no such results have been reported before in the literature. Figure 12 shows the current waveform of the $\mathrm{BC}$ for two temperature values. A decrement in terms of amplitude can be observed when a decrement of temperature occurs.

\section{CONCLUSIONS}

In this paper, the mechanoelectrical transduction properties of a BC-based sensing element were investigated. More specifically, the investigation analysed the dependence of the transduction properties on the geometry of the system and the environmental temperature.

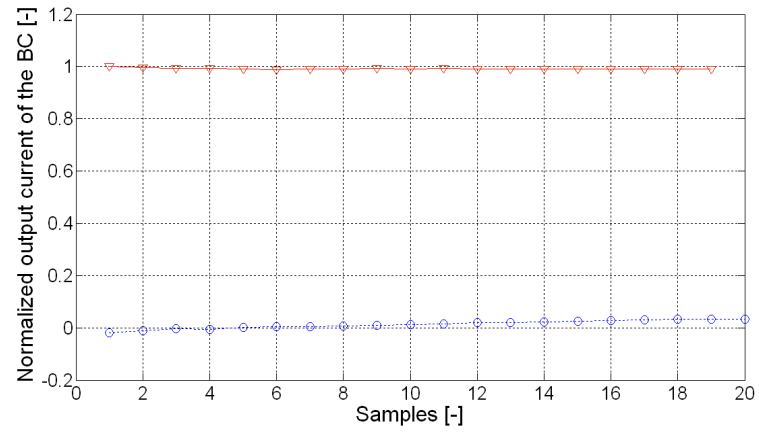

Figure 11. Generated current value as a function of number of samples. The analysis includes two values of temperature, $5{ }^{\circ} \mathrm{C}$ (blue diamonds) and $35^{\circ} \mathrm{C}$ (red triangles), respectively. Normalisation has been conducted with respect to the maximum value of the signal.

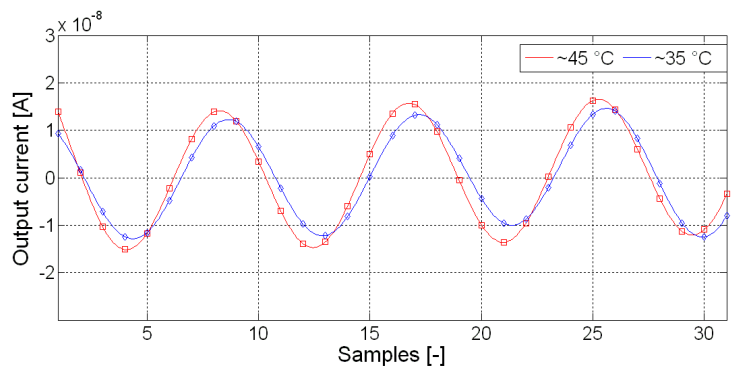

Figure 12. Generated current waveform as a function of the acquired samples. The analysis includes two temperature values, $45^{\circ} \mathrm{C}$ (squares) and $35{ }^{\circ} \mathrm{C}$ ( diamonds), respectively. 
Reported results evince for concluding that the geometry of the system can be used to modulate the expected output signal.

Temperature was revealed to have a strong influence on the system's transducing properties. More specifically, reported results showed that the dependence on the temperature can be explained by the active role of mobile charges inside the composite: as a matter of fact, a reduction of the working temperature below the melting point of the IL virtually annulled the sensing signal.

\section{ACKNOWLEDGEMENT}

This research has been partially funded by the University of Catania within the project's Piani della Ricerca Dipartimentale.

\section{REFERENCES}

[1] Y. H. Jung, T. H. Chang, H. Zhang, C. Yao, Q. Zheng, V. W. Yang, H. Jiang, High-performance green flexible electronics based on biodegradable cellulose nanofibril paper, Nature Communications 6 (2015), p. 7170.

DOI: https://doi.org/10.1038/ncomms 8170

[2] M. C. Vats, S. K. Singh, E-waste characteristic and its disposal. Am Assoc Sci and Techno 1(2) (2014), pp. 49-61. Online [Accessed 04 December 2020]

http://article.aascit.org/file/pdf/9040718.pdf

[3] J. Kim, Y. B. Seo, Electro-active paper actuators, Smart Materials and Structures 11(3) (2002), p. 355.

DOI: https://doi.org/10.1088/0964-1726/11/3/305

[4] J. Kim, S. Yun, Z. Ounaies, Discovery of cellulose as a smart material, Macromolecules 39(12) (2006), pp. 4202-4206. DOI: https://doi.org/10.1021/ma060261e

[5] D. Nevstrueva, K. Murashko, V. Vunder, A. Aabloo, A. Pihlajamäki, M. Mänttäri, J. Torop, Natural cellulose ionogels for soft artificial muscles, Colloids and Surfaces B: Biointerfaces 161 (2018), pp.244-251.

DOI: https://doi.org/10.1016/j.colsurfb.2017.10.053

[6] C. H. Je, K. J. Kim, Cellophane as a biodegradable electroactive polymer actuator, Sensors and Actuators A: Physical 112(1) (2004), pp. 107-115.

DOI: https://doi.org/10.1016/i.sna.2003.11.005

[7] European Union, SETIS - Strategic Energy Technologies Information System, Online [Accessed 30 October 2020] https://setis.ec.europa.eu/technologies/energy-intensiveindustries/energy-efficiency-and-co2-reduction-in-the-pulppaper-industry/info

[8] E. Núñez-Carmona, A. Bertuna, M. Abbatangelo, V. Sberveglieri, E. Comini, G. Sberveglieri, BC-MOS: The novel bacteria cellulose based MOS gas sensors, Materials Letters 237 (2019), pp. 69-71.

DOI: https://doi.org/10.1016/i.matlet.2018.11.011

[9] S. Goswami, G. R. Dillip, S. Nandy, A. N. Banerjee, A. Pimentel, S. W. Joo, R. Martins, E. Fortunato, Biowaste-derived carbon black applied to polyaniline-based high-performance supercapacitor microelectrodes: Sustainable materials for renewable energy applications, Electrochimica Acta 316 (2019), pp. 202-218.

DOI: https://doi.org/10.1016/i.electacta.2019.05.133

[10] F. Wang, Z. Jin, S. Zheng, H. Li, S. Cho, H. J. Kim, S. Park, Highfidelity bioelectronic muscular actuator based on porous carboxylate bacterial cellulose membrane, Sensors and Actuators
B: Chemical 250 (2017), pp. 402-411.

DOI: https://doi.org/10.1016/i.snb.2017.04.124

[11] H. Hosseini, M. Kokabi, S. M. Mousavi, Conductive bacterial cellulose/multiwall carbon nanotubes nanocomposite aerogel as a potentially flexible lightweight strain sensor, Carbohydrate Polymers 201 (2018), pp. 228-235.

DOI: https://doi.org/10.1016/i.carbpol.2018.08.054

[12] G. Di Pasquale, S. Graziani, A. Pollicino, C. Trigona, Green inertial sensors based on bacterial cellulose, Proc. of IEEE Sensors Applications Symposium 2019(SAS), Sophia Antipolis, France, 11-13 March 2019, pp. 1-4. DOI: https://doi.org/10.3390/proceedings2019015036

[13] G. Di Pasquale, S. Graziani, A. Pollicino, C. Trigona, "Paper" based sensor for deformation measurements. Proc. of 2019 IEEE International Instrumentation and Measurement Technology Conference (I2MTC), Auckland, New Zealand, 20-23 May 2019, pp. 1-5.

DOI: https://doi.org/10.1109/I2MTC.2019.8826962

[14] G. Di Pasquale, S. Graziani, A. Pollicino, C. Trigona, A bacterial cellulose based mass sensor, Proc. of 2019 IEEE International Symposium on Measurements \& Networking $(M \& N)$, Catania, Italy, 8-10 July 2019, pp. 1-4. DOI: https://doi.org/10.1109/IWMN.2019.8805008

[15] G. Di Pasquale, S. Graziani, A. Licciulli, R. Nisi, A. Pollicino, C. Trigona, Geometrical analysis of a bacterial cellulose-based sensing element, Proc. of 2019 II Workshop on Metrology for Industry 4.0 and IoT (MetroInd4. 0\&IoT), Naples, Italy, 4-6 June 2019, pp. 225-228.

DOI: https://doi.org/10.1109/METROI4.2019.8792875

[16] P. Brunetto, L. Fortuna, P. Giannone, S. Graziani, S. Strazzeri, Static and dynamic characterization of the temperature and humidity influence on IPMC actuators, IEEE Transactions on Instrumentation and Measurement 59(4) (2009), pp. 893-908. DOI: https://doi.org/10.1109/TIM.2009.2026613

[17] P. Brunetto, L. Fortuna, P. Giannone, S. Graziani, S. Strazzeri, Characterization of the temperature and humidity influence on ionic polymer-metal composites as sensors, IEEE Transactions on Instrumentation and Measurement 60(8) (2011), pp. 29512959

DOI: https://doi.org/10.1109/TIM.2011.2118870

[18] C. Chen, B.Y. Liu, Changes in major components of tea fungusmetabolites during prolonged fermentation, Journal of appliedmicrobiology 89 (2000), pp. 834-839.

DOI: https://doi.org/10.1046/j.1365-2672.2000.01188.x

[19] S. Pal, R. Nisi, M. Stoppa, A. Licciulli, Silver-functionalized bacterial cellulose as antibacterial membrane for wound-healing applications, ACS Omega 2(7) (2017), pp. 3632-3639.

DOI: https://doi.org/10.1021/acsomega.7b00442

[20] Sigma-Aldrich, Catalogue. Online [Accessed 30 October 2020] https://www.sigmaaldrich.com/catalog/product/aldrich/90077 2?lang=it\&region $=$ IT

[21] A. Olivares, I. Cosme, S. Mansurova, A. Kosarev, H. E. Martinez, Study of electrical conductivity of PEDOT:PSS at temperatures $>$ $300 \mathrm{~K}$ for hybrid photovoltaic applications, Proc. of $12^{\text {th }}$ International Conference on Electrical Engineering, Computing Science and Automatic Control (CCE), Mexico City, Mexico 2830 October 2015, pp. 1-3. DOI: https://doi.org/10.1109/ICEEE.2015.7357906

[22] S.-S. Kim, J. H. Jeon, C.-D. Kee, I.-H. Oh, Electro-active hybrid actuators based on freeze-dried bacterial cellulose and PEDOT:PSS, Smart Materials and Structures 22(8) (2013) art. no. 085026, 9 pages.

DOI: https://doi.org/10.1088/0964-1726/22/8/085026 